\title{
Wiggles in the cosmic microwave background radiation: Echoes from nonsingular cyclic inflation
}

\author{
Tirthabir Biswas, ${ }^{1,2}$ Anupam Mazumdar, ${ }^{2,3}$ and Arman Shafieloo ${ }^{4}$ \\ ${ }^{1}$ Department of Physics, St. Cloud State University, St. Cloud, Minnesota 56301, USA \\ ${ }^{2}$ Department of Physics, Loyola University, New Orleans, Louisiana 70118, USA \\ ${ }^{3}$ Physics Department, Lancaster University, Lancaster, LA1 4YB, United Kingdom \\ ${ }^{4}$ Physics Department, University of Oxford, Oxford, OX1 3NP, United Kingdom
}

(Received 13 May 2010; published 17 December 2010)

\begin{abstract}
In this paper we consider a unique model of inflation where the universe undergoes rapid asymmetric oscillations, each cycle lasting $\sim 10^{6}$ Planck times. Over many, many cycles the space-time expands to mimic the standard inflationary scenario. Moreover, these rapid oscillations leave a distinctive periodic signature in $\ln k$ in the primordial power spectrum, where $k$ denotes the comoving scale. Although the cyclic-inflation model contains additional parameters as compared to the standard power-law spectrum, the improvement to the fit of the 7-year WMAP data is significant.
\end{abstract}

DOI: 10.1103/PhysRevD.82.123517

PACS numbers: 98.80.Cq, 98.80.Bp, 98.80.Qc

\section{INTRODUCTION}

Primordial inflation has been very successful in explaining the perturbations in the cosmic microwave background $(\mathrm{CMB})$ radiation and the large-scale structures of the universe [1]; for a recent review see [2]. However, inflation has some outstanding problems - in particular, it does not encode a nonsingular geodesically complete evolution [3]. We will present a unique singularity free geodesically complete realization of inflation in the context of cyclic cosmologies.

In cyclic cosmological models, rather than having a singular beginning of time, our universe can be made eternal in both past and future [4-13]. However, in most cyclic scenarios the effort has been to produce primordial fluctuations within a single long cycle. ${ }^{1}$ Although there has been progress [15-17], this has proved rather challenging in comparison to the success which inflation enjoys in explaining the observed near scale-invariant perturbations in the CMB.

In this paper we provide a simple alternative to the standard inflation and cyclic universe scenarios in the form of a cyclic-inflation model which tries to incorporate the successes of both; our model includes a nonsingular cyclic phase of evolution where in every cycle the universe contracts a little less than it expands, leading to an overall growth. In fact, over many, many cycles the space-time resembles that of inflation. Thus, in close analogy with inflation, we can explain how the seed perturbations generated at much higher energy densities can be stretched to the observable scales, and why the spectrum is nearly scale free. Additionally, the model leaves distinct signatures of the rapid oscillations the universe undergoes by modifying the power spectrum with periodic signatures. Last but not the least, it turns out that the cyclic-inflationary phase requires a negative potential energy, but the universe can gracefully exit to a positive potential region marking the

\footnotetext{
${ }^{1}$ Recently, there have been attempts to calculate how perturbations can evolve through various cycles [14].
}

end of the inflationary phase and the onset of a standard radiation dominated era. Thus our model may provide a way of reaching a positive energy vacuum from a plethora of negative energy vacua in the string landscape [18]. Finally, Tolman's entropy problem (which is equivalent to the problem of geodesic incompleteness in our model) can be naturally addressed by including a preinflationary emergent phase where the scale factor starts to oscillate periodically as we approach infinity; the size of the universe never becomes vanishingly small [10].

Let us consider a simple cyclic-inflation model, where the universe is mostly dominated by radiation, and the cycles are (approximately) periodic in energy densities. This follows if, first, we assume that quantum gravitational effects trigger a bounce whenever some critical Planckian energy density is reached. Second, we need a - ve cosmological constant (CC), $-\Lambda$, which ensures that the universe turns around and starts to recollapse once the radiation energy density dilutes and becomes equal to $\Lambda$. Thus contrary to common expectations, in the presence of matter the universe does not get stuck in anti-de Sitter vacua $[12,19]$, but rather starts to cycle. These cycles are typically short, the time period, $\tau=\alpha M_{p} / \sqrt{\Lambda}$, where $\alpha \sim \mathcal{O}(1)$ and $M_{p}=2.4 \times 10^{18} \mathrm{GeV}$ [12]. We shall show that in order to obtain the correct amplitude of CMB fluctuations we will require $\Lambda$ to be close to the conventional string/grand unified theory scale, $\Lambda^{1 / 4} \sim 10^{-3} M_{p}$, so that $\tau \sim 10^{6} M_{p}^{-1}$.

Now, provided there is exchange of energy between radiation and some other forms of matter, then, as a natural consequence of the second law of thermodynamics, one expects the cycles to be asymmetric. The total entropy in the universe increases monotonically, and by the same factor in every cycle: $S_{n+1} / S_{n} \equiv 1+3 \kappa$. Since entropy is proportional to the volume this means that if, for instance, we track the scale factor at the bounce point of consecutive cycles, then

$$
a_{n+1} / a_{n}=1+\kappa \text { for } \kappa \ll 1 \text {. }
$$


While the above scenario can be realized in many different ways, here we will consider a simple toy model with two species, massless radiation and some massive particle which interact with each other. It is clear from (1) that, over many asymmetric cycles, the evolution of the universe looks very similar to that of ordinary inflation with an average Hubble expansion rate $H_{\mathrm{av}}=\kappa / \tau$. We will see later that this "cyclic-inflationary" phase can indeed address the usual cosmological puzzles such as isotropy, horizon, flatness and homogeneity.

What about the spectrum of the primordial fluctuations? To match the COBE normalization, the power spectrum associated with metric fluctuations must be given by $\mathcal{P}_{\Phi} \sim 10^{-10}$. Now in general, since matter couples very weakly to gravity, in the sub-Hubble phase (when the wavelength of a given comoving mode is smaller than the cosmological time scale), when the metric fluctuations are generated from the matter fluctuations, the amplitude is suppressed by the Planck scale; see Appendix B for a detailed derivation. Typically we have

$$
\mathcal{P}_{\Phi} \propto k^{3} \Phi_{k}^{2} \sim \rho / M_{p}^{4} \sim 10^{-10} .
$$

Once the wavelength becomes larger than the cosmological time scale, the metric fluctuations effectively freeze at the value of $\rho$ when the particular mode crosses the Hubble radius. This intuitive picture will essentially let us argue why the perturbations in our model will have a near scaleinvariant spectrum with a distinctive periodic feature (in $\ln k$ ) which, in fact, provides a significant improvement in the WMAP 7-yr fit, and hence may be detectable in the future experiments. The two most important parameters governing the physics are $\Lambda$ and $\kappa$. While the former determines the amplitude of fluctuations, the latter characterizes the wiggles on top of the near scale-invariant spectrum.

Finally, let us discuss the graceful exit problem in this inflationary scenario. If we are stuck in a $-v e \mathrm{CC}$, then the above inflationary phase persists forever and one can never obtain a universe like ours. Fortunately, one can exit the inflationary phase if instead of a negative cosmological constant we have a dynamical scalar field whose potential interpolates between a negative and a positive

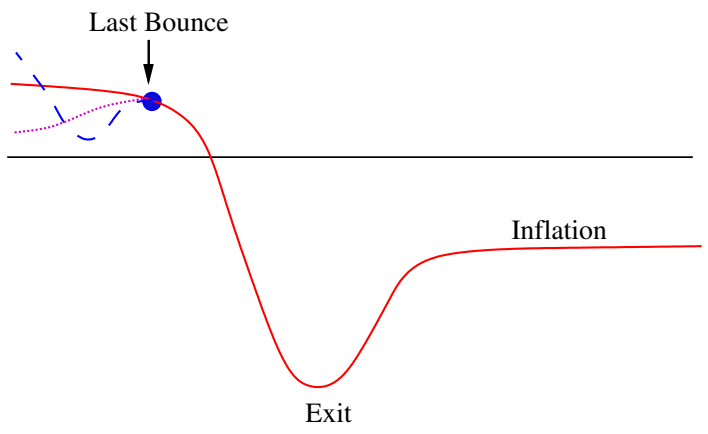

FIG. 1 (color online). Typical potentials: In the positive energy side three different possibilities are consistent with our model. cosmological constant as depicted in Fig. 1. Since $V(\phi) \rightarrow$ $-\Lambda$ as $\phi \rightarrow \infty$, we can realize the inflationary phase, but the scalar field keeps rolling towards smaller $\phi$ and eventually there comes a (last) cycle; in this last contraction phase the scalar field gains enough energy to zoom through the minimum and reach the + ve CC phase.

The paper is organized as follows: In Sec. II, we describe our toy model and the background evolution which mimics the inflationary space-time. Next, in Sec. III, we explain why we expect to get a nearly scale-invariant spectrum with characteristic small wiggles in our model. We also try to fit our model with the WMAP data and report our findings. In IV, we end with a discussion of the standard cosmological puzzles in the context of our model and an outlook towards future research directions.

\section{THE MODEL AND BACKGROUND EVOLUTION}

\section{A. The cycles}

Let us start by looking at a simple toy model where we have a negative cosmological constant, and the "matter content" of the universe consists of a single nonrelativistic species along with relativistic degrees of freedom. The scenario we will present here is very similar to what was considered in [12], except that we will have a radiation dominated universe, whereas in [12] the dominant contribution to energy density always came from the nonrelativistic species. The advantage of having a radiation dominated universe is that the model can then easily avoid the black-hole overproduction problem, common in cyclic models, as the Jeans length is always large and comparable to the Hubble length. Most of the analysis in the present radiation dominated scenario closely parallels what was carried out in [12], only some numerical factors change. Hence, we will keep this part of the discussion brief, and refer the readers to the appendixes for details.

To obtain the cyclic evolution, we are going to make a couple of assumptions: First, we will assume that during contraction if a critical Planckian energy density, $\rho_{b}$, is reached, the universe bounces back nonsingularly to a phase of expansion. ${ }^{2}$ We will see later that the details of the mechanism of the bounce is not particularly important for our model. Second, we are going to demand that the relativistic and the nonrelativistic species remain in thermal equilibrium above a certain critical temperature, $T_{c}$, but below this temperature the massive nonrelativistic degrees of freedom fall out of equilibrium, and consequently when they decay into radiation, thermal entropy is generated. We will see shortly that this is the crucial ingredient that makes our cycles slightly asymmetric and causes the universe to "effectively" inflate.

\footnotetext{
${ }^{2}$ Several different bouncing models have been considered in the literature, and in most models a bounce occurs when the energy density reaches close to the Planck density; see for instance $[9,16,20,21]$
} 
In the nonthermal phase the Hubble equation reads

$$
H^{2}=\frac{T_{c}^{4}}{3 M_{p}^{2}}\left(\frac{\Omega_{r}}{a^{4}}+\frac{\Omega_{m}}{a^{3}}-\frac{\Lambda}{T_{c}^{4}}\right),
$$

where $-\Lambda \equiv-M_{s}^{4}$ is the negative cosmological constant, and the $\Omega$ 's are related to the energy densities via

$$
\rho_{m}=T_{c}^{4} \frac{\Omega_{m}}{a^{3}} \quad \text { and } \quad \rho_{r}=T_{c}^{4} \frac{\Omega_{r}}{a^{4}} .
$$

For definiteness, we consider a closed compact universe ${ }^{3}$ with a volume $V \equiv T_{c}^{-3} a^{3}$. Although spatial curvature plays no role in the cyclic-inflationary phase because it is inflated away very quickly, the advantage of having a closed universe is that before the inflationary phase, one can have an emergent phase [10] when the spatial curvature is important and this phase can solve the problem of geodesic incompleteness that standard inflationary cosmology is plagued with. We will elaborate on this in the concluding section.

Let us define the ratio of the equilibrium energy densities of the nonrelativistic (massive) and relativistic (massless) species at the transition point, $T=T_{c}$, to be given by

$$
\mu \equiv \frac{\rho_{m, c}}{\rho_{r, c}} .
$$

We will here be interested in scenarios where $\mu \ll 1$, so that to a good approximation the universe is always radiation dominated. In order to understand the dynamics, it is instructive to first look into the evolution when nonrelativistic and relativistic species are noninteracting. Then $\Omega$ 's would just be constants yielding a Friedmann-RobertsonWalker cosmology, and we will have a cyclic universe scenario. Let us start tracking the evolution just as the universe enters the nonthermal phase at the transition temperature, $T_{c}$, during the expanding branch. As the universe expands further, the temperature decreases until $T \sim \Lambda^{1 / 4}$ at which point the universe turns around due to the presence of the negative cosmological constant. As it contracts the temperature rises. Once the temperature crosses $T_{c}$, the universe enters the thermal (still contracting) phase. Once Planckian densities are reached, according to our prior assumption, a quantum bounce transitions the universe back to the expanding branch. Once more the temperature dilutes, and as it falls below $T_{c}$, the next cycle begins. One can compute the time period of these cycles just by integrating the Hubble equation (3). In Appendix A we have obtained the expression in the approximation where we neglect the nonrelativistic matter contribution to the Hubble equation (3) (see also [12]): ${ }^{4}$

\footnotetext{
${ }^{3}$ For an open or a flat universe one just has to rephrase all the arguments in terms of entropy density rather than the total entropy and volume of the universe.

${ }^{4} \mathrm{We}$ have ignored the time spent in the thermal bounce phase, because approximately it is given by $\tau_{b} \sim M_{p} / T_{c}^{2}$, which is much shorter than $\tau$ as long as $T_{c}^{4} \gg \Lambda$.
}

$$
\tau \approx \frac{\sqrt{3} \pi M_{p}}{2 \sqrt{\Lambda}} .
$$

Importantly, this is a constant and does not change from cycle to cycle as it does not depend on $\Omega_{r}, \Omega_{m}$ (as we will see shortly, the $\Omega$ 's will increase from cycle to cycle with the gradual expansion of the universe). Another way to think about this is that although the cycles are asymmetric in scale factor, it is periodic in energy densities, and it is the various energy densities that the Hubble rate is sensitive to.

\section{B. Energy exchange and inflation}

Let us now turn our attention to the overall growth of the scale factor, which is best expressed in terms of entropy growth from cycle to cycle. The amount by which the entropy grows as matter gets converted into radiation depends on the details of the microphysical processes involved, but for us the only thing that is important to realize is that since the different $\rho$ 's and $H(t)$ are periodic functions of time, this growth in entropy density will not change from cycle to cycle. For the purpose of illustration in Appendix A, we considered a very simple scenario where in the nonthermal phase the nonrelativistic species can simply decay into the radiative degrees. If the decay time is much larger than the time period of the cycle, $\Gamma \tau \ll 1$, one can analytically compute the increase in the scale factor in a given cycle:

$$
\kappa \approx \frac{1.6 \mu \Gamma M_{p} T_{c} g^{1 / 4}}{\Lambda^{3 / 4}}+\mathcal{O}\left(\mu^{2}\right) .
$$

As expected the increase in entropy depends on the various mass scales involved, the parameter $\mu$ which controls how much of nonrelativistic matter is present in the fluid, and $\Gamma$ which determines how fast the massive particles decay into radiation. It is easy to see that by choosing appropriate parameter values one can make this number small.

Now, in a more realistic paradigm one will have several species involved. A stringy model could involve a thermal Hagedorn phase near the bounce when the massive string states are kept in thermal equilibrium with the massless degrees [10,12], but below some critical temperature when the relevant scattering cross sections become too small, they would fall out of equilibrium. If these modes consequently decay into radiation, entropy would be produced in a manner very similar to what we discussed above. After the turnaround, as the universe starts to contract and the energy density increases, the massive states can be replenished from radiation by scattering processes. In this case the parameter $\kappa$ would capture stringy physics involving thermodynamics, scattering cross sections and decay rates.

Finally, let us comment briefly on the graceful exit mechanism. Once we replace the negative $\mathrm{CC}$ with a negative potential of the form depicted in Fig. 1, the universe can exit the cyclic-inflationary mechanism. This is possible because if the slope of the potential suddenly 
becomes steep, the total scalar energy density can become positive during a single contraction phase. Once the total scalar energy density is positive, since the energy density can only increase in the contracting phase, the scalar field cannot turn back in the negative potential region (at the turning point this would imply negative total energy). This is a well-known result; see for instance $[12,19]$. The universe bounces one last time when the energy density reaches $\sim \rho_{b}$. As long as the bounce occurs when the scalar field is already in, or is "sufficiently near," the positive part of the potential, the present universe will emerge dynamically with a positive cosmological constant after the graceful exit from the cycling inflationary phase. Note that the universe cannot turn around any more as the scalar energy density is no longer negative. Moreover, after the bounce since the kinetic energy of the scalar field starts to redshift as $a^{-6}$, even if it dominates matter/radiation, it will quickly become subdominant, ensuring the entry into a matter/ radiation dominated universe.

Approximate calculations corroborating the above argument were presented in [12], and we are currently performing a detailed numerical exploration [22] of the entire parameter space to determine whether any fine-tuning is required for the mechanism to succeed.

\section{CMB SPECTRUM}

\section{A. Scale-invariant envelope with periodic modulations}

Let us first provide a general argument as to why we expect to obtain a nearly scale-invariant spectrum in our model. We are interested in tracking $\Phi_{k}$. As the scale factor evolves, so does the physical wavelength, $\lambda_{k}(t)$. Now the evolution of $\Phi_{k}$ at a given physical wavelength, $\lambda$, depends on the background environment that the mode "sees"; i.e., it depends on the different energy densities involved, $\left\{\rho_{i}(t)\right\}$, where $i$ labels the various fluid components. Thus another way of looking at the problem is to realize that the evolution of $\Phi_{k}$ depends on the curves $\left\{\rho_{i}(\lambda)\right\}$, and these curves will in general be different for different comoving modes. For simplicity, let us first consider the case when we have a - ve $\mathrm{CC}$ (then cycles are precisely periodic in energy densities), and look at two modes which are related by $\kappa$, the factor by which the universe expands in a given cycle:

$$
k^{\prime}=(1+\kappa) k \Rightarrow(1+\kappa) \lambda_{k^{\prime}}=\lambda_{k} .
$$

The curves $\left\{\rho_{i}(\lambda)\right\}$ for these two modes are completely identical, the $k^{\prime}$ mode lagging behind a cycle as compared to the $k$ mode. Therefore as one evolves from $\lambda \rightarrow 0$, the sub-Hubble phase, to $\lambda \rightarrow \infty$, the super-Hubble phase where the fluctuations freeze, these two modes should change by the same factor. If we use the traditional Bunch-Davis vacuum initial conditions at $t \rightarrow-\infty$ (or $\lambda \rightarrow 0$ ) like in standard inflation, then the sub-Hubble metric power spectrum is scale free: $\Phi_{k} \rightarrow k^{-3 / 2}$ (see Appendix B for details, and [23] for a review). According to our previous arguments therefore, as perturbations become super-Hubble $(t \rightarrow \infty$ and $\lambda \rightarrow \infty)$, this scaleinvariance should be preserved, modulo periodic deviations:

$$
k^{3}\left|\Phi_{k}\right|^{2}=k^{\prime 3}\left|\Phi_{k^{\prime}}\right|^{2} \Rightarrow \mathcal{P}_{\Phi}(\ln k+\ln (1+\kappa))=\mathcal{P}_{\Phi}(\ln k) .
$$

Since $\kappa$ is typically a small number in our model, we expect to have a near scale-invariant spectrum.

Let us actually try to calculate the spectrum by making a few simple assumptions. First, we are going to assume that as long as wavelengths of fluctuations are smaller than the cosmological time scale their power spectrum is determined by the energy density of the fluid, vis-à-vis (2). Second, we will assume that once the modes become super-Hubble they stop evolving and freeze out. These are indeed what one finds in general relativity (GR), and as we will see, in our scenario the freezeout occurs near the turnaround, away from the non-GR bounce. Hence we expect these assumptions to hold true but we do want to caution the readers about possible modifications coming from the non-GR bouncing phase.

Having said that, let us go ahead and try to calculate the amplitude of perturbations, which essentially boils down to calculating the amplitude at the time of the "last exit." To determine when this last exit occurs let us first look at the cosmological time scale. In the usual monotonically expanding universe this is just given by the Hubble radius. However, during the bounce the time scale, $\tau_{b}$ is determined by the bounce energy density, $\rho_{b}$. Typically

$$
\tau_{b}=\beta \frac{M_{p}}{\sqrt{\rho_{b}}},
$$

where $\beta \sim \mathcal{O}(1)$ is constant. The cosmological time scale thus approximately behaves as follows: During the bounce phase it stays a constant and is given by $\tau_{b},(10)$. After the bounce, radiation dominated GR takes over; the cosmological scale just corresponds to the Hubble scale, and starts to increase. Near the turnaround phase, it reaches its maximum value which is essentially the time period of the cycle determined by the cosmological constant [see (6)]. After the turnaround, the cosmological scale decreases back to its minimum value near the bounce, and this cycle repeats itself (see Fig. 2).

In the mean time, the physical scale corresponding to a particular comoving perturbation keeps oscillating with the cycles of the universe, but because of the asymmetry gradually expands. Thus, there is an initial "pure sub-Hubble" phase where the fluctuations are always smaller than the cosmological scale. Then there appears a "mixed phase" where the fluctuations go in and out of the "Hubble radius." Finally, there comes a last cycle where the wavelength goes out of the Hubble radius never to return (see Fig. 2). We call this the last exit. According to the two assumptions we have made, all we have to do is to calculate the amplitude of fluctuations of the modes at their last exit point. 


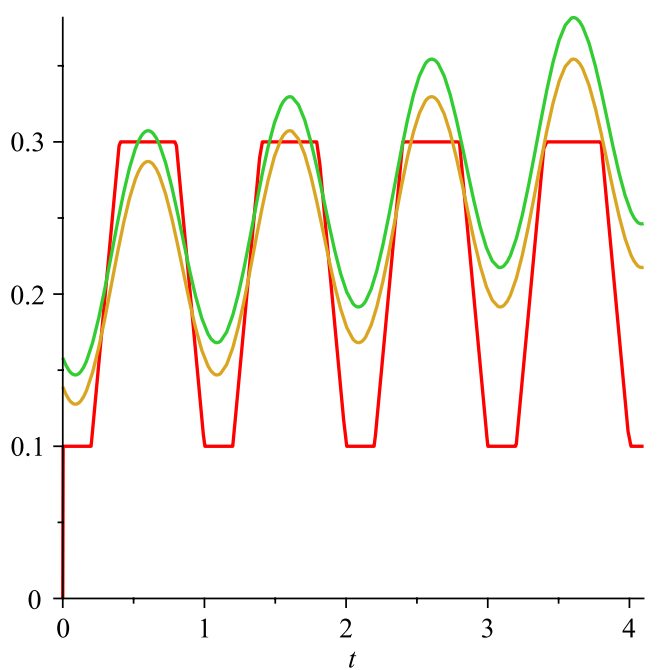

FIG. 2 (color online). The last exit: The darkest (red) curve corresponds to the cosmological time scale, while the other curves [the top (green) and the bottom (yellow) shaded curves] represent the physical wavelengths of two different comoving modes. The two modes are initially in a mixed phase but then they make their last exits after which they evolve as superHubble modes. While the green wavelength exits in the third cycle, the yellow curve has to wait for another cycle to make its last exit. The two modes experience identical background cosmology, the yellow curve only lagging behind by a cycle as compared to the green curve.

For this purpose let us focus on a single cycle. Consider modes which are super-Hubble at the bounce, i.e., $\lambda_{k} \gtrsim \tau_{b}$. After the bounce in the radiation dominated expansion phase, the Hubble radius grows faster than the scale factor, and hence these modes start to reenter the Hubble radius. This process continues till we reach the turnaround point when the Hubble radius stops growing and therefore the modes stop reentering. After the turnaround in the radiation dominated contraction phase, precisely the opposite happens; i.e., the modes start to exit the Hubble radius. Now, and this is the key point, since in our model the universe contracts a little less than it expands, those modes which are the first to exit the Hubble radius in the contraction phase never come back inside the Hubble radius in the following cycle. They have just made their last exit after which their amplitudes freeze. This is illustrated in Fig. 2.

We are now ready to calculate the spectrum. To this end we need to compute the energy density of radiation at the time the modes exit during contraction. The radiation density is, as usual, given by

$$
\rho_{r}=\rho_{b}\left(\frac{a}{a_{b}}\right)^{-4}
$$

To obtain the spectral dependence we need to use the Hubble crossing condition

$$
\lambda_{k}=a / k=H^{-1} \Rightarrow k \propto(-t)^{-(1 / 2)},
$$

since during the radiation-dominated contraction, $H \propto 1 / t$, and $a \propto \sqrt{-t}$. Thus we have

$$
\rho_{r} \propto(-t)^{-2} \propto k^{4} .
$$

If $k_{1}$ corresponds to the comoving wave which is the first one to exit the Hubble radius during contraction, then all the modes with values up to $k_{1}(1+\kappa)$ also exit the mixed phase in the same cycle. Thus the power spectrum for the modes that exit the mixed phase in a given cycle reads

$$
\mathcal{P}_{\Phi}=\gamma \frac{\Lambda}{M_{p}^{4}}\left(\frac{k}{k_{1}}\right)^{4} \quad \text { for } k_{1}<k<(1+\kappa) k_{1},
$$

$\gamma$ being an $\mathcal{O}(1)$ parameter relating the power spectrum to the energy density of the fluid; we note that since the modes exit near the turnaround the energy density in radiation, which controls the amplitude (2), must be close to $\Lambda$. Now, once the wave number increases by a factor $(1+\kappa)$, the fluctuations can no longer exit the mixed phase because they reenter the Hubble radius during the following expansion. They therefore must wait for the next cycle for their last exit (see Fig.2), and consequently the power spectrum repeats.

\section{B. Spectral tilt}

The fact that the energy density of the scalar field during the inflationary phase is not a constant but slowly evolves implies that the spectral envelope (without the periodic wiggles) is not going to be precisely scale invariant but have a tilt. Now we are working on the assumption that the amplitude of oscillation freezes when the physical wavelength of a given comoving mode equals the cosmological time scale. This in turn depends at what scale the turnaround occurs. In our scenario, the turnaround occurs when the positive radiation density cancels the negative scalar field density. Thus, $\mathcal{P}_{\Phi} \propto\left|\rho_{\phi}\right|$, and the power spectrum will change slowly as $\phi$ evolves.

Now, if $V^{\prime}(\phi)=0, \phi$ would just get Hubble damped and freeze. However, for a nonzero $V^{\prime}(\phi)$ it will evolve in a manner very similar to the inflationary slow roll: $3 H_{a v} \dot{\phi}=$ $-V^{\prime}(\phi)$, so that

$$
\rho_{\phi}=V(\phi)+\frac{\dot{\phi}^{2}}{2} \approx V(\phi)+\frac{V^{\prime 2}(\phi) \alpha^{2} M_{p}^{2}}{18 V(\phi) \kappa^{2}} .
$$

Depending upon the parameters of the potential it is possible for $\rho(\phi)$ to either increase or decrease as $\phi$ evolves, resulting in a red or a blue tilt, respectively. For instance, if our potential in the inflating region is modeled as $V(\phi)=$ $-\Lambda\left(1+e^{-\nu \phi / M_{p}}\right)$, with $e^{-\nu \phi / M_{p}} \ll 1$, then one finds

$$
\rho_{\phi} \approx-\Lambda\left[1+e^{-\nu \phi / M_{p}}-\left(\nu^{2} \alpha^{2} / 18 \kappa^{2}\right) e^{-2 \nu \phi / M_{p}}\right] .
$$

Since $\kappa$ is a small parameter one can check that it is possible for the third term to dominate over the second so that we obtain a red tilt. We plan to investigate the detail dynamics in future - the important point for now is that, in 
general, we will have a nonzero spectral tilt which can be either red or blue. Thus we expect the power spectrum to have the following approximate form:

$$
\mathcal{P}_{\Phi}=\gamma \frac{\rho_{b}}{M_{p}^{4}}\left(\frac{k}{k_{1}}\right)^{\eta_{s}-1} f(k),
$$

where $f(k)$ is defined as

$$
\begin{aligned}
f(k) & =\left(\frac{k}{k_{1}}\right)^{4} \quad \text { for } k_{1}<k<(1+\kappa) k_{1} \quad \text { and } \\
f(k(1+\kappa)) & =f(k) .
\end{aligned}
$$

In particular, a distinctive feature of these modulations is that $f$ varies from 1 to $1+4 \kappa$ as the wave number goes from $k_{1}$ to $(1+\kappa) k_{1}$. In other words, the amplitude of fluctuations, $\Delta f=2 \kappa$, is related to the period of fluctuations, $\Delta \ln k=\kappa$, in a simple way. This is important since previously very different physical models have been considered which give similar oscillatory modulations of the spectrum, but the amplitude and the period are typically two independent parameters [24].

\section{Fitting WMAP-7yr data}

To estimate the effect of the wiggles as a good approximation to the discontinuous wiggles (18), one can consider a smooth oscillatory modulation since it has almost the same shape and oscillatory behavior and is also more appropriate to be used to calculate the angular power spectrum using CAMB or COSMOMC. We can therefore use a power spectrum of the form

$$
\mathcal{P}_{\Phi}=\frac{\Lambda}{3 M_{p}^{4}}\left(\frac{k}{k_{0}}\right)^{\eta_{s}-1}\left[1+2 \kappa \cos \left(\frac{2 \pi}{\kappa} \ln \frac{k}{k_{0}}+\theta\right)\right]
$$

where $k_{0}$ is an arbitrary normalization scale. The main additional parameter as compared to the standard primordial spectrum is $\kappa<1 / 2$, although we also have an additional phase factor in $\theta \in[0,2 \pi]$.

In order to see how well the cyclic inflation fits the CMB data, we have done some coarse sampling in the parameter space. For baryonic abundance, $\Omega_{b} h^{2}=0.0228$, cold dark matter abundance, $\Omega_{\mathrm{dm}} h^{2}=0.1156, h=0.70$, the optical depth, $\tau=0.082$, and parameters of the primordial spectrum of $n_{s}=0.963, \kappa=0.0455$ and $\theta=2.546$ (radian), we found a very good likelihood of $-2 \ln (L)=-7470.5$ to 7-year WMAP data [1].

In Fig. 3, we see the resultant best fit angular power spectrum $(t t$ and $t e$ ) from the cyclic model of inflation. In comparison with the best fit power-law form of the primordial spectrum and power law with running spectral tilt model, our model can improve the likelihood by $\Delta \chi^{2}=$ -3.9 and $\Delta \chi^{2}=-2.7$, respectively. It is interesting that in comparison with the phenomenological model of power law with running, our model has a distinctively better likelihood, although the models have a similar number of degrees of freedom. Note here that we have assumed a

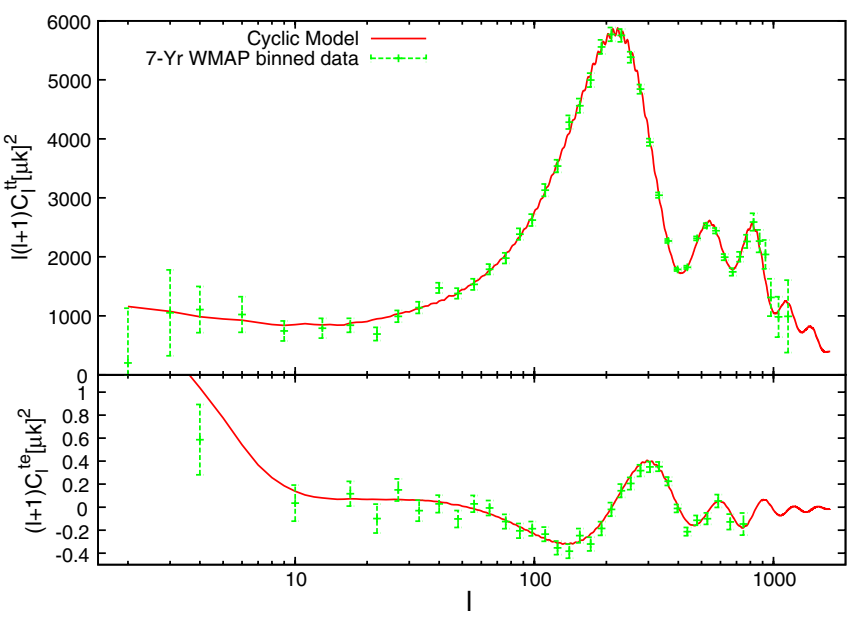

FIG. 3 (color online). Resultant best fit $C_{l}^{t t}$ (top panel) and $C_{l}^{t e}$ (bottom panel) from the cyclic inflation in comparison with 7-year WMAP binned data.

fixed value of $k_{0}=0.05 \mathrm{Mpc}^{-1}$ in Eq. (19). In comparison with the simple power-law form of the primordial spectrum, considering the additional free parameters in our theory, the improvement in the likelihood is not as big as to claim that our model is preferred by the data [25], but at the same time, the improvement in the likelihood is not insignificant. Allowing tensor modes in the analysis will not improve the best fit likelihood and we expect a very small value of $r$ (tensor to scaler ratio). We will leave a proper cosmological parameter estimation using $\mathrm{CMB}$ and other cosmological data for future publication, but the derived likelihood and the best fit parameters already suggest that this model should provide a good fit.

\section{THE STANDARD COSMOLOGICAL PUZZLES AND FUTURE OUTLOOK}

Before we conclude, let us look at the problem of growth of inhomogeneity/overproduction of black holes. The matter fluctuations, $\delta \equiv \delta \rho / \rho$, can only grow as long as their wavelengths are larger than the Jeans length, $\lambda_{J}$, given by $\lambda_{J} \sim c_{s} M_{p} / \sqrt{\rho}$ where $c_{s}^{2} \equiv \partial p / \partial \rho$ is the sound velocity square. Now, in our scenario the cycles are short, and most of the energy content is in radiation so that the sound speed is very close to the speed of light, and accordingly $\lambda_{J} \sim H^{-1} \sim \tau$. In other words, the sub-Hubble fluctuations do not grow. On the other hand, once the wavelengths become larger than the cosmological time scale, $\tau$, they become super-Hubble fluctuations and evolve according to the Poisson equation: $\delta_{k}=k^{2} \Phi_{k} /\left(a^{2} \rho\right)$, where $\Phi_{k}$ is the Newtonian potential characterizing the metric perturbations. Now in the super-Hubble phase, $\Phi_{k}$, becomes a constant while $\rho$ oscillates between a minimum and a maximum energy density. Thus we have $\delta_{k}<$ $k^{2} \Phi_{k} /\left(a^{2} \rho_{\min }\right) \sim k^{2} \Phi_{k} M_{p} /\left(a^{2} \sqrt{\Lambda}\right)$. In other words $\delta_{k}$ falls as $a^{-2}$ in the super-Hubble phase just as in ordinary 
inflation. Finally, cyclic cosmologies are notoriously plagued with mixmaster chaotic behavior as one approaches the big crunch/bounce, since anisotropies grow as $\sim a^{-6}$. However, in our model the same reasoning that resolves the flatness problem also solves this issue. Once the cyclic-inflationary phase is "activated" in a small patch of the universe the chaotic mixmaster behavior is avoided in subsequent cycles as the universe becomes more and more isotropic due to the overall growth of the scale factor.

Finally, it is clear that the cyclic-inflationary phase cannot be "past-eternal," it will suffer from the same geodesic incompleteness problem as the standard inflationary models, and in the context of cyclic models this is referred to as Tolman's entropy problem [4]. Fortunately, it was demonstrated in [10] that once one includes spatial curvature into the story this problem is naturally solved as long as the universe is closed: Before the cyclicinflationary phase, the universe undergoes an "emergent" phase where as time goes to - ve infinity the scale factor asymptotes to a periodic (in time) cyclic evolution. In this phase, the turnaround occurs when radiation density cancels the - ve curvature density. The universe does grow slowly through the course of entropy production in the cycles and at some point the curvature density becomes less negative than the scalar potential energy and one enters the cyclic-inflationary phase. This mechanism is actually similar to the "emergent universe" scenario proposed in the context of standard inflation [26].

To summarize we have presented a unique way of realizing inflation within the cyclic universe scenario, where the particle trajectory is geodesically complete in either past or future. Although in each cycle the universe expands only a little bit, one can obtain a large number of total inflationary e-foldings, $\mathcal{N}_{\text {tot }}$, over many, many cycles. As long as $\mathcal{N}_{\text {tot }} \geq 60$ it is clear that we can explain the horizon and the flatness problems. Furthermore, every cycle leaves its quantum imprint in the CMB with potentially observable wiggles determined by the enhancement of the scale factor during every oscillation.

\section{ACKNOWLEDGMENTS}

A.M. and A.S. acknowledge the support of the EU Network UniverseNet (MRTN-CT-2006-035863) and thank Jan Hamann and Xingang Chen for useful discussions.

\section{APPENDIX A: $\tau$ AND $\Delta S$}

Here we calculate the approximate time period and the increase in entropy in a given cycle. We will calculate this under the approximation that the dust component can be neglected as $\mu \ll 1$ and treat $\Omega$ as a constant as the amount of matter decay in a given cycle is negligible.

To calculate the time period we start by rewriting the Hubble equation as

$$
d t=\frac{d a}{\dot{a}}=\frac{\sqrt{3} M_{p} d a}{T_{c}^{2} a \sqrt{\frac{\Omega_{r}}{a^{4}}-\frac{\Lambda}{T_{c}^{4}}}}
$$

so that the time period is given by

$$
\tau \approx \frac{2 \sqrt{3} M_{p}}{T_{c}^{2}} \int_{a_{c}}^{a_{T}} \frac{d a}{a \sqrt{\frac{\Omega_{r}}{a^{4}}-\frac{\Lambda}{T_{c}^{4}}}},
$$

where we have neglected the duration in the thermal bounce phase as it is going to be much shorter than the above integral. Now the turnaround scale factor is given by

$$
a_{T}=\left(\frac{\Omega_{r} T_{c}^{4}}{\Lambda}\right)^{1 / 4} .
$$

Thus the above integral can be reexpressed as

$$
\tau=\frac{2 \sqrt{3} M_{p}}{\sqrt{\Lambda}} \int_{a_{c}}^{a_{T}} \frac{d a}{a \sqrt{\left(\frac{a_{T}}{a}\right)^{4}-1}}=\frac{2 \sqrt{3} M_{p}}{\sqrt{\Lambda}} \int_{\epsilon}^{1} \frac{y d y}{\sqrt{1-y^{4}}},
$$

where $\epsilon \equiv a_{c} / a_{T} \ll 1$. Thus we have

$$
\tau \approx \frac{2 \sqrt{3} M_{p}}{\sqrt{\Lambda}} \int_{0}^{1} \frac{y d y}{\sqrt{1-y^{4}}}=\frac{\sqrt{3} \pi M_{p}}{2 \sqrt{\Lambda}} .
$$

In a similar manner we can proceed to calculate the approximate entropy increase. Phenomenologically, energy exchanges can be captured by generalizing conservation equations $[8,10,27]$ for the two fluids to

$$
\dot{\rho}_{r}+4 H \rho_{r}=T_{c}^{4} s, \quad \dot{\rho}_{m}+3 H \rho_{m}=-T_{c}^{4} s
$$

which now includes an energy exchange term. We can easily compute the net entropy increase [12]:

$\dot{S}=\dot{S}_{r}+\dot{S}_{m}=a^{3} s\left(\frac{3 b_{r} T_{c}}{4 \rho_{r}^{1 / 4}}-\frac{T_{c}}{M}\right)=a^{3} s\left(\frac{T_{c}}{T_{r}}-1\right)$,

where we have used the usual expressions for the thermodynamic entropies associated with matter and radiation,

$$
S_{r}=\frac{4 \rho_{r} V}{3 T}=\frac{4}{3} g^{1 / 4} \Omega_{r}^{3 / 4}, \quad S_{m}=\frac{\rho_{m} V}{M}=\Omega_{m} .
$$

Here $M \approx T_{c}$ corresponds to the mass of the nonrelativistic particles, and in our convention, $g=\left(\pi^{2} / 30\right) g_{*}$, where $g_{*}$ is the number of "effective" massless degrees of freedom.

The growth of the entropy in a given cycle will obviously depend on the phenomenological function $s$. For the simplest case, which captures the physics of the decay of the nonrelativistic species into radiation, $s$ is given by

$$
s=\frac{\Gamma \rho_{m}}{T_{c}^{4}},
$$

$\Gamma$ being the decay rate. One can now obtain the entropy increase in a given cycle by simultaneously solving for the Hubble equation (3), the continuity equations (A6) and entropy growth (A7). For some special cases this can 
also be done analytically. For the purpose of illustration we are going to look at the case when the decay time is much larger than the time period of the cycle, $\Gamma \tau \ll 1$. This means that basically the $\Omega$ 's change very little over a given cycle and for the purpose of estimating the entropy increase from (A8) we can treat them to be constants. We can approximate $e^{-\Gamma t} \approx 1$ in the expression for $\dot{S}$. Using (A7), (3), (A9), and (4) we find

$$
\frac{d S}{d a}=\frac{\sqrt{3} \Gamma \Omega_{m} M_{p}}{a \sqrt{\Lambda}} \frac{\left(\frac{a}{a_{c}}-1\right)}{\sqrt{\left(\frac{a_{T}}{a}\right)^{4}-1}} .
$$

Thus the increase in entropy in a given cycle can be calculated as

$$
\begin{aligned}
\Delta S & =\frac{2 \sqrt{3} \Gamma \Omega_{m} M_{p}}{\sqrt{\Lambda}} \int_{a_{c}}^{a_{T}} \frac{d a}{a} \frac{\left(\frac{a}{a_{c}}-1\right)}{a \sqrt{\left(\frac{a_{T}}{a}\right)^{4}-1}} \\
& =\frac{2 \sqrt{3} \Gamma \Omega_{m} M_{p}}{\sqrt{\Lambda}} \int_{y_{c}}^{1} d y \frac{y\left(\frac{y}{y_{c}}-1\right)}{\sqrt{1-y^{4}}} \\
& \approx \frac{2 \sqrt{3} \Gamma \Omega_{m} M_{p}}{\sqrt{\Lambda} y_{c}} \int_{0}^{1} d y \frac{y^{2}}{\sqrt{1-y^{4}}} .
\end{aligned}
$$

Now, we know that at the transition temperature the energy density in radiation must be given by

$$
\rho_{r}=g T_{c}^{4}=T_{c}^{4} \frac{\Omega_{r}}{a_{c}^{4}} \Rightarrow a_{c}=\left(\frac{\Omega_{r}}{g}\right)^{1 / 4} .
$$

Using the expression for $a_{T}$ then we find

$$
y_{c}=\frac{a_{c}}{a_{T}}=\frac{\Lambda^{1 / 4}}{g^{1 / 4} T_{c}} .
$$

Using the expressions for energy density and entropy we can also easily relate $\Omega_{m}$ with the total entropy $S$ :

$$
\Omega_{m} \approx \frac{3 \mu S}{4} .
$$

Thus we finally have

$$
\Delta S \approx \frac{3 \sqrt{3} \mu \Gamma S M_{p}}{2 \sqrt{\Lambda} y_{c}} \int_{0}^{1} d y \frac{y^{2}}{\sqrt{1-y^{4}}} \approx \frac{1.6 \mu \Gamma S M_{p} T_{c} g^{1 / 4}}{\Lambda^{3 / 4}} .
$$

\section{APPENDIX B: AMPLITUDE OF THE PERTURBATIONS}

The discussion below is completely general and can be applied for any hydrodynamical fluid with $\omega \in(0,1)$, although in our model since we have a radiation dominated universe the appropriate choice is $\omega=1 / 3$. A very similar analysis can also be done for scalar fields [23].

It is assumed that the universe starts in a vacuum state in the infinite past when all the fluctuations are sub-Hubble. It is well-known that the appropriate quantum variable that one should use to obtain the "initial conditions" is the Mukhanov variable $v$ which obeys the following field equation [23]:

$$
v^{\prime \prime}-c_{s}^{2} \nabla^{2} v-\frac{z^{\prime \prime}}{z} v=0,
$$

where

$$
z \equiv \frac{a \sqrt{\beta}}{-\mathcal{H} c_{s}} \quad \text { and } \quad \beta \equiv \mathcal{H}^{2}-\mathcal{H}^{\prime} .
$$

$c_{s}$ is the velocity of sound defined by

$$
c_{s}^{2} \equiv \frac{\partial \rho}{\partial p}=\omega
$$

for an ideal fluid. For a hydrodynamical fluid, $z$ simplifies to

$$
z=-a \sqrt{\frac{3(1+\omega)}{2 \omega}}
$$

The evolution equation for $v$ thus simplifies to

$$
v_{k}^{\prime \prime}+\omega k^{2} v_{k}-\frac{q(q-1)}{\tau^{2}} v_{k}=0 .
$$

The above equation can be solved exactly in terms of the Bessel functions:

$$
\begin{aligned}
v_{k}= & \sqrt{-\tau}\left[V_{1 k} J_{q-(1 / 2)}(-\sqrt{\omega} k \tau)\right. \\
& \left.+V_{2 k} J_{-q+(1 / 2)}(-\sqrt{\omega} k \tau)\right] .
\end{aligned}
$$

To determine $V_{k}$ 's we need to look at the sub-Hubble limit of $v_{k}$ as $-\tau \rightarrow \infty$. Using the asymptotic expansion,

$$
J_{\nu}(x) \rightarrow \sqrt{\frac{2}{\pi x}} \cos \left(x-\frac{\nu \pi}{2}-\frac{\pi}{4}\right),
$$

we have

$$
\begin{aligned}
v_{k} \approx & \sqrt{\frac{2}{\pi \sqrt{\omega} k}}\left[V_{1 k} \cos \left(-\sqrt{\omega} k \tau+\frac{q \pi}{2}-\frac{\pi}{2}\right)\right. \\
& \left.+V_{2 k} \cos \left(-\sqrt{\omega} k \tau-\frac{q \pi}{2}\right)\right] .
\end{aligned}
$$

In other words, provided $\omega>0, v_{k}$ approaches the oscillatory function, as one would naturally expect in the Minkowski vacuum state in the sub-Hubble limit as $\tau \rightarrow-\infty$. One can compute the quantum fluctuations associated with a given $k$ mode in the quantum vacuum state:

$$
v(x)=\int d^{3} k\left(a_{k} e^{i(k \cdot x-\sqrt{\omega} k t)}+a_{k}^{*} e^{-i(k \cdot x-\sqrt{\omega} k t)}\right) .
$$

It is known that the number operator $\left\langle\left|a_{k}\right|^{2}\right\rangle \sim 1 / k$. Implementing the quantum vacuum fluctuations as initial conditions then leads to the conclusion that

$$
V_{1 k} \sim V_{2 k} \sim \mathcal{O}(1)
$$


constants, independent of $k$. Thus we recover the wellknown result that $v_{k} \sim k^{-1 / 2}$ in the sub-Hubble phase.

Our next task is to relate the Mukhanov variable $v$ to $\Phi$. For the hydrodynamical fluid, the relationship is given by

$$
v \equiv \sqrt{\omega}\left(u^{\prime}+\mathcal{H} u\right)
$$

where

$$
u=\frac{2 M_{p}^{2} \Phi}{\sqrt{(1+\omega) \rho}} .
$$

After some straightforward algebra, using (B6), (B11), and (B12), one finds that

$$
\begin{aligned}
\Phi_{k}= & \frac{\sqrt{(1+\omega)(-\tau) \rho}}{2 M_{p}^{2} k}\left[V_{1} J_{q+(1 / 2)}(-\sqrt{\omega} k \tau)\right. \\
& \left.+V_{2} J_{-q-(1 / 2)}(-\sqrt{\omega} k \tau)\right] .
\end{aligned}
$$

Actually, it is easier to substitute (B13) in (B12) and then (B11) to check that this leads to (B6), keeping in mind the following identity:

$$
J_{\nu}^{\prime}(z)=J_{\nu-1}(z)-\frac{\nu J_{\nu}(z)}{z} .
$$

One can verify that the above solution indeed satisfies the GR perturbation equation

$$
\Phi_{k}^{\prime \prime}+\frac{6(1+\omega)}{\tau(1+3 \omega)} \Phi_{k}^{\prime}+\omega k^{2} \Phi_{k}=0 .
$$

Let us look at the sub-Hubble limit of (B13):

$$
\begin{aligned}
\Phi_{k}= & k^{-(3 / 2)} \sqrt{\frac{(1+\omega) \rho}{2 \pi \sqrt{\omega} M_{p}^{4}}}\left[V_{1} \cos \left(-\sqrt{\omega} k \tau+\frac{q \pi}{2}\right)\right. \\
& \left.+V_{2} \cos \left(-\sqrt{\omega} k \tau-\frac{q \pi}{2}-\frac{\pi}{2}\right)\right] .
\end{aligned}
$$

Thus we find that the amplitude of oscillations evolves with the energy density of the background and is suppressed by the Planck energy density. Thus these vacuum fluctuations are extremely tiny at low energy densities. The dimensionless power spectrum is thus proportional to

$$
\mathcal{P}_{\Phi, \text { sub }} \equiv k^{3}\left\langle\left|\Phi_{k}\right|^{2}\right\rangle \propto \frac{\rho}{M_{p}^{4}} .
$$

[1] E. Komatsu et al., arXiv:1001.4538 [Astrophys. J. Suppl. Ser. (to be published)].

[2] A. Mazumdar and J. Rocher, arXiv:1001.0993.

[3] A. D. Linde, D. A. Linde, and A. Mezhlumian, Phys. Rev. D 49, 1783 (1994); A. Borde and A. Vilenkin, Phys. Rev. Lett. 72, 3305 (1994); A. Borde, A. H. Guth, and A. Vilenkin, Phys. Rev. Lett. 90, 151301 (2003); T. Vachaspati and M. Trodden, Phys. Rev. D 61, 023502 (1999).

[4] R. C. Tolman, Phys. Rev. 37, 1639 (1931).

[5] H. Bondi and T. Gold, Mon. Not. R. Astron. Soc. 108, 252 (1948); F. Hoyle, Mon. Not. R. Astron. Soc. 108, 372 (1948); J. Astrophys. Astron. 28, 67 (2007).

[6] A. A. Starobinsky, Phys. Lett. 91B, 99 (1980).

[7] J. Khoury, B. A. Ovrut, P. J. Steinhardt, and N. Turok, Phys. Rev. D 64, 123522 (2001); P. J. Steinhardt and N. Turok, Phys. Rev. D 65, 126003 (2002); Science 296, 1436 (2002).

[8] J.D. Barrow, D. Kimberly, and J. Magueijo, Classical Quantum Gravity 21, 4289 (2004).

[9] M. G. Brown, K. Freese, and W. H. Kinney, J. Cosmol. Astropart. Phys. 03 (2008) 002; L. Baum and P. H. Frampton, Phys. Rev. Lett. 98, 071301 (2007).

[10] T. Biswas, arXiv:0801.1315; T. Biswas and S. Alexander, Phys. Rev. D 80, 043511 (2009).

[11] F. T. Falciano, M. Lilley, and P. Peter, Phys. Rev. D 77, 083513 (2008).

[12] T. Biswas and A. Mazumdar, Phys. Rev. D 80, 023519 (2009).
[13] Y. F. Cai and E. N. Saridakis, J. Cosmol. Astropart. Phys. 10 (2009) 020.

[14] R. H. Brandenberger, Phys. Rev. D 80, 023535 (2009); J. Zhang, Z. G. Liu, and Y.S. Piao, arXiv:1007.2498; Y.S. Piao, Phys. Lett. B 691, 225 (2010).

[15] E. I. Buchbinder, J. Khoury, and B. A. Ovrut, Phys. Rev. D 76, 123503 (2007); J. L. Lehners, P. McFadden, N. Turok, and P. J. Steinhardt, Phys. Rev. D 76, 103501 (2007); J. Khoury and P. J. Steinhardt, Phys. Rev. Lett. 104, 091301 (2010).

[16] T. Biswas, A. Mazumdar, and W. Siegel, J. Cosmol. Astropart. Phys. 03 (2006) 009; T. Biswas, R. Brandenberger, A. Mazumdar, and W. Siegel, J. Cosmol. Astropart. Phys. 12 (2007) 011.

[17] Y.F. Cai, T.t. Qiu, R. Brandenberger, and X.m. Zhang, Phys. Rev. D 80, 023511 (2009).

[18] M. R. Douglas and S. Kachru, Rev. Mod. Phys. 79, 733 (2007); H. Firouzjahi, S. Sarangi, and S. H. H. Tye, J. High Energy Phys. 09 (2004) 060.

[19] G. N. Felder, A. V. Frolov, L. Kofman, and A. V. Linde, Phys. Rev. D 66, 023507 (2002).

[20] T. Biswas, T. Koivisto, and A. Mazumdar, arXiv:1005.0590 [J. Cosmol. Astropart. Phys. (to be published)].

[21] M. Bojowald, Phys. Rev. Lett. 86, 5227 (2001); A. Ashtekar, T. Pawlowski, P. Singh, and K. Vandersloot, Phys. Rev. D 75, 024035 (2007); A. Ashtekar, T. Pawlowski, and P. Singh, Phys. Rev. Lett. 96, 141301 (2006); Phys. Rev. D 74, 084003 (2006); G. 
Date and G. M. Hossain, Phys. Rev. Lett. 94, 011302 (2005).

[22] T. Biswas, T. Koivisto, and A. Mazumdar (in progress).

[23] V.F. Mukhanov, H. A. Feldman, and R. H. Brandenberger, Phys. Rep. 215, 203 (1992).

[24] G. F. R. Ellis and R. Maartens, Classical Quantum Gravity 21, 223 (2004); G. F. R. Ellis, J. Murugan, and C. G. Tsagas, Classical Quantum Gravity 21, 233 (2004).
[25] H. Peiris and L. Verde, Phys. Rev. D 81, 021302 (2010); J. Hamann, A. Shafieloo, and T. Souradepp, J. Cosmol. Astropart. Phys. 04 (2010) 010.

[26] J. Martin and C. Ringeval, Phys. Rev. D 69, 083515 (2004); R. Flauger, L. McAllister, E. Pajer, A. Westphal, and G. Xu, J. Cosmol. Astropart. Phys. 06 (2010) 009.

[27] J. D. Barrow and M. P. Dabrowski, Mon. Not. R. Astron. Soc. 275, 850 (1995). 